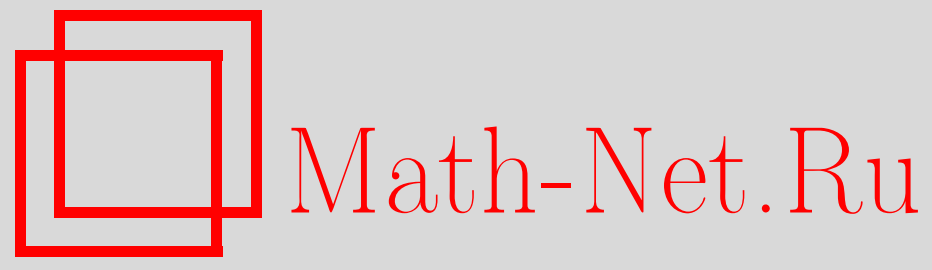

Н. Н. Попов, О. О. Чернова, Метод решения задачи о чистом сдвиге стохастически неоднородной плоскости в условиях установившейся ползучести, Becmн. Сам. гос. техн. ун-та. Сер. Физ.-мат. науки, 2012, выпуск 4(), 97-105

DOI: https://doi.org/10.14498/vsgtu1132

Использование Общероссийского математического портала Math-Net.Ru подразумевает, что вы прочитали и согласны с пользовательским соглашением

http: //www . mathnet.ru/rus/agreement

Параметры загрузки:

IP : 54.166 .219 .16

26 апреля 2023 г., 08:42:43

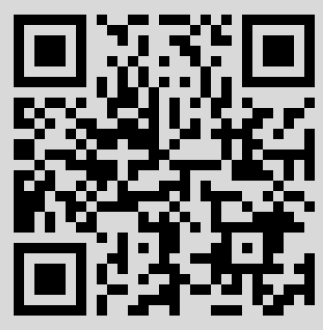




\title{
МЕТОД РЕШЕНИЯ ЗАДАЧИ О ЧИСТОМ СДВИГЕ СТОХАСТИЧЕСКИ НЕОДНОРОДНОЙ ПЛОСКОСТИ В УСЛОВИЯХ УСТАНОВИВШЕЙСЯ ПОЛЗУЧЕСТИ
}

\author{
Н. Н. Попов, О.О. Чернова \\ Самарский государственный технический университет, \\ 443100, Россия, Самара, ул. Молодогвардейская, 244. \\ E-mails: ponick25@gmail.com, chernova_olga@citydom.ru
}

Разработан аналитический метод решения нелинейной задачи установившейся ползучести при чистом сдвиге стохастически неоднородной плоскости на основе второго приближения метода малого параметра. Введено ограничение о малости упругих дебормаций, которьми допустимо пренебречь. Стохастичность введена в определяющие соотношения ползучести, взятье в соответствии с нелинейной теорией вязкого течения, при помощи случайной однородной бункиии координат. С исполъзованием метода разложения компонент тензора напряжений по малому параметру до членов второго порядка малости получена система дифберенииалъных уравнений в частных производных относительно напряжений для первого и второго приближений. Решение системы строилосъ путём введения функиии напряжений. Вычислены математическое ожсдание и дисперсии случайного поля напряжений. Проведён анализ результатов, полученных в первом и втором приближениях.

Ключевые слова: чистый сдвиг, метод малого параметра, установившаяся ползучесть, второе приближение, стохастическая задача.

Наиболее часто используемым методом решения стохастических краевых задач является метод малого параметра, который позволяет свести статистически нелинейную задачу к последовательности статистически линейных задач. Следует отметить, что данный подход связан с трудностями вычислительного характера, поэтому при решении нелинейных стохастических задач обычно ограничиваются первым (реже вторым) приближением метода малого параметра. Метод малого параметра детально разработан лишь для стохастических задач линейной теории упругости В. А. Ломакиным $[1,2]$. Его применение в теории ползучести сдерживается проблемами не только статистической, но и физической нелинейности.

В данной работе приводится решение нелинейной задачи установившейся ползучести при чистом сдвиге стохастически неоднородной плоскости на основе второго приближения метода малого параметра. Аналогичная задача в корреляционном приближении (соответствует первому приближению метода малого параметра) на основе метода спектрального представления рассматривалась в работе [3]. Вводится ограничение о малости упругих деформаций и считается, что ими допустимо пренебречь. Деформируемый материал считается стохастически неоднородным, так что компоненты тензоров напряжений и скоростей деформаций установившейся ползучести в декартовой ортогональной системе координат являются случайными функциями

Николай Николаевич Попов (к.ф.-м.н., доц.), доцент, каф. прикладной математики и информатики.

Ольга Олеговна Чернова, младший научный сотрудник, каф. прикладной математики и информатики. 
координат $x_{1}$ и $x_{2}$.

Определяющие соотношения ползучести принимаются в соответствии с нелинейной теорией вязкого течения (установившейся ползучести) в стохастической форме [4]

$$
\dot{p}_{i j}=c s^{n-1}\left(\sigma_{i j}-\frac{1}{3} \delta_{i j} \sigma_{k k}\right)\left(1+\alpha U\left(x_{1}, x_{2}\right)\right),
$$

где $s^{2}=0,5\left(3 \sigma_{i j} \sigma_{i j}-\sigma_{i i} \sigma_{j j}\right)$ - интенсивность напряжений, $\dot{p}_{i j}-$ компоненты тензора деформаций, $\sigma_{i j}$ - компоненты тензора напряжений, $\delta_{i j}-$ символ Кронекера, $U\left(x_{1}, x_{2}\right)$ - случайная однородная функция, описывающая возмущение реологических свойств материала с математическим ожиданием $\langle U\rangle=0$ и дисперсией $\left\langle U^{2}\right\rangle=1 ; c, n-$ постоянные материала, $\alpha-$ число, определяющее степень неоднородности материала. По повторяющимся индексам производится суммирование от 1 до 2. В дальнейшем предполагается, что $\alpha-$ малый параметр, по которому производится разложение в ряд (метод малого параметра).

$\mathrm{K}$ определяющим соотношениям ползучести (1) присоединяются уравнения равновесия для напряжений и условие совместности деформаций, сформулированное для скоростей деформаций ползучести:

$$
\begin{gathered}
\sigma_{i j, j}=0, \quad i, j=1,2, \\
\Lambda_{i j} \Lambda_{k l} \dot{p}_{j k, i l}=0 .
\end{gathered}
$$

Здесь $\Lambda=\left(\begin{array}{rr}0 & 1 \\ -1 & 0\end{array}\right)$ - единичный антисимметричный псевдотензор.

Соотношения (1)-(3) задают стохастическую задачу ползучести, которая решается относительно напряжений на основе второго приближения метода малого параметра.

После подстановки определяющего соотношения (1) в уравнение совместности (2) и сокращения на величину $\left(s^{2}\right)^{(n-5) / 2}$ в работе [5] было получено следующее уравнение:

$$
\begin{aligned}
\left(\frac{n-1}{2} \frac{n-3}{2}\left(s_{, 2}^{2}\right)^{2}(1+\alpha U)+\frac{n-1}{2} s^{2} s_{, 22}^{2}(1+\alpha U)+\right. & \\
& \left.+(n-1) s^{2} s_{, 2}^{2} \alpha U,_{2}+\left(s^{2}\right)^{2} \alpha U, 22\right)\left(2 \sigma_{11}-\sigma_{22}\right)+ \\
+ & 2\left(\frac{n-1}{2} s^{2} s_{, 2}^{2}(1+\alpha U)+\left(s^{2}\right)^{2} \alpha U, 2\right)\left(2 \sigma_{11}-\sigma_{22}\right)_{, 2}+ \\
& +\left(s^{2}\right)^{2}(1+\alpha U)\left(2 \sigma_{11}-\sigma_{22}\right),{ }_{22}+ \\
+ & \frac{n-1}{2} \frac{n-3}{2}\left(s_{, 1}^{2}\right)^{2}(1+\alpha U)+\frac{n-1}{2} s^{2} s_{,{ }_{11}}(1+\alpha U)+ \\
& \left.+(n-1) s^{2} s_{,}^{2} \alpha U,{ }_{1}+\left(s^{2}\right)^{2} \alpha U,{ }_{11}\right)\left(2 \sigma_{22}-\sigma_{11}\right)+ \\
+ & 2\left(\frac{n-1}{2} s^{2} s_{,{ }_{1}}^{2}(1+\alpha U)+\left(s^{2}\right)^{2} \alpha U,{ }_{1}\right)\left(2 \sigma_{22}-\sigma_{11}\right),{ }_{1}+
\end{aligned}
$$




$$
\begin{gathered}
+\left(s^{2}\right)^{2}(1+\alpha U)\left(2 \sigma_{22}-\sigma_{11}\right),{ }_{11}- \\
-3(n-1)\left(\frac{n-3}{2} s_{, 1}^{2} s_{, 2}^{2}(1+\alpha U)+s^{2} s_{, 12}^{2}(1+\alpha U)+\right. \\
\left.+s^{2} s_{, 2}^{2} \alpha U,{ }_{1}+s^{2} s_{, 1}^{2} \alpha U,,_{2}\right) \sigma_{12}-6\left(s^{2}\right)^{2} \alpha U,_{12} \sigma_{12}- \\
-6\left(\frac{n-1}{2} s^{2} s_{,{ }_{1}}{ }_{2}(1+\alpha U)+\left(s^{2}\right)^{2} \alpha U,{ }_{1}\right) \sigma_{12,2}- \\
-6\left(\frac{n-1}{2} s^{2} s_{, 2}{ }_{2}(1+\alpha U)+\left(s^{2}\right)^{2} \alpha U,{ }_{2}\right) \sigma_{12,1}- \\
-6\left(s^{2}\right)^{2}(1+\alpha U) \sigma_{12,12}=0 .
\end{gathered}
$$

Для приближённого решения уравнения (4) используется метод разложения компонент тензора напряжений $\sigma_{i j}$ и интенсивности напряжений $s^{2}$ по малому параметру $\alpha$ до членов второго порядка малости:

$$
\begin{gathered}
\sigma_{i j}=\sigma_{i j}^{0}+\alpha \sigma_{i j}^{1}+\alpha^{2} \sigma_{i j}^{2}, \quad\left\langle\sigma_{i j}\right\rangle=\sigma_{i j}^{0}, \\
s^{2}=s_{0}^{2}+\alpha s_{1}+\alpha^{2} s_{2} .
\end{gathered}
$$

В силу того, что в условии чистого сдвига детерминированные нормальные напряжения $\sigma_{11}^{0}$ и $\sigma_{22}^{0}$ равны нулю, а касательное напряжение $\sigma_{12}^{0}$ является постоянным, имеем

$$
s_{0}^{2}=3 \sigma_{12}^{0}, \quad s_{1}=6 \sigma_{12}^{0} \sigma_{12}^{1}, \quad s_{2}=\left(\sigma_{11}^{1}\right)^{2}+\left(\sigma_{22}^{1}\right)^{2}-\sigma_{11}^{1} \sigma_{22}^{1}+3\left(\sigma_{12}^{1}\right)^{2}+6 \sigma_{12}^{0} \sigma_{12}^{2} .
$$

Уравнение (4) путём подстановки в него представлений (5), (6) и приравнивания коэффициентов при одинаковых степенях $\alpha$ приводится к системе двух уравнений:

$$
\left(2 \sigma_{11}^{1}-\sigma_{22}^{1}\right), 22+\left(2 \sigma_{22}^{1}-\sigma_{11}^{1}\right),{ }_{11}-6 \sigma_{12,12}^{1}-6 k s_{1,12} \sigma_{12}^{0}=6 U,{ }_{12} \sigma_{12}^{0},
$$

$$
\begin{aligned}
& \left(2 \sigma_{11}^{2}-\sigma_{22}^{2}\right),{ }_{22}+\left(2 \sigma_{22}^{2}-\sigma_{11}^{2}\right),{ }_{11}-6 \sigma_{12,12}^{2}-6 k s_{2},{ }_{12} \sigma_{12}^{0}= \\
& =6 k q s_{1,1} s_{1,2} \sigma_{12}^{0}+6 k\left(s_{1,12} \sigma_{12}^{1}+s_{1,1} \sigma_{12,2}^{1}+s_{1,2} \sigma_{12,1}^{1}-\right. \\
& \left.-\frac{s_{1} s_{1},{ }_{12} \sigma_{12}^{0}}{s_{0}^{2}}+s_{1,2} U,{ }_{1} \sigma_{12}^{0}+s_{1,1} U,{ }_{2} \sigma_{12}^{0}\right)-k\left(s_{1,22}\left(2 \sigma_{11}^{1}-\sigma_{22}^{1}\right)+s_{1,11}\left(2 \sigma_{22}^{1}-\sigma_{11}^{1}\right)+\right. \\
& \left.+2 s_{1,2}\left(2 \sigma_{11}^{1}-\sigma_{22}^{1}\right),_{2}+2 s_{1,{ }_{1}}\left(2 \sigma_{22}^{1}-\sigma_{11}^{1}\right),{ }_{1}\right)-U,{ }_{22}\left(2 \sigma_{11}^{1}-\sigma_{22}^{1}\right)-2 U,_{2}\left(2 \sigma_{11}^{1}-\sigma_{22}^{1}\right),{ }_{2}- \\
& -U,{ }_{11}\left(2 \sigma_{22}^{1}-\sigma_{11}^{1}\right)-2 U,_{1}\left(2 \sigma_{22}^{1}-\sigma_{11}^{1}\right),_{1}+6 U,_{12} \sigma_{12}^{1}-6 U U,_{12} \sigma_{12}^{0},
\end{aligned}
$$

где $k=(n-1) /\left(2 s_{0}^{2}\right), q=(n-3) /\left(2 s_{0}^{2}\right)$.

Подставив в уравнения (7), (8) приближения $s_{1}, s_{2}$ интенсивности напряжений $s^{2}$, получим систему дифференциальных уравнений в частных производных относительно $\sigma_{i j}^{1}$ и $\sigma_{i j}^{2}$ :

$$
2 \sigma_{11,22}^{1}-\sigma_{22,22}^{1}+2 \sigma_{22,11}^{1}-\sigma_{11,11}^{1}-6 n \sigma_{12,12}^{1}=6 \sigma_{12}^{0} U,{ }_{12},
$$




$$
\begin{aligned}
& 2 \sigma_{11,22}^{2}-\sigma_{22,22}^{2}+2 \sigma_{22}^{2},{ }_{11}-\sigma_{11,11}^{2}-6 n \sigma_{12,12}^{2}=216 k q \sigma_{12,1}^{1} \sigma_{12,2}^{1}\left(\sigma_{12}^{0}\right)^{3}+ \\
& \quad+36 k \sigma_{12}^{0}\left(2 \sigma_{12,1}^{1} \sigma_{12,2}^{1}-\sigma_{12}^{1} \sigma_{12,12}^{1}+\sigma_{12,2}^{1} U,_{1} \sigma_{12}^{0}+\sigma_{12,{ }_{1}}^{1} U,_{2} \sigma_{12}^{0}\right)- \\
& -6 k \sigma_{12}^{0}\left(\sigma_{12,22}^{1}\left(2 \sigma_{11}^{1}-\sigma_{22}^{1}\right)+\sigma_{12,11}^{1}\left(2 \sigma_{22}^{1}-\sigma_{11}^{1}\right)+2 \sigma_{12,2}^{1}\left(2 \sigma_{11,2}^{1}-\sigma_{22,2}^{1}\right)+\right. \\
& \left.+2 \sigma_{12,{ }_{1}}^{1}\left(2 \sigma_{22,1}^{1}-\sigma_{11,1}^{1}\right)-r,{ }_{12}\right)-U,{ }_{22}\left(2 \sigma_{11}^{1}-\sigma_{22}^{1}\right)-2 U,_{2}\left(2 \sigma_{11,2}^{1}-\sigma_{22,2}^{1}\right)- \\
& \quad-U,{ }_{11}\left(2 \sigma_{22}^{1}-\sigma_{11}^{1}\right)-2 U,_{1}\left(2 \sigma_{22,1}^{1}-\sigma_{11,1}^{1}\right)+6 U,{ }_{12} \sigma_{12}^{1}-6 U U,{ }_{12} \sigma_{12}^{0},
\end{aligned}
$$

где $r=\left(\sigma_{11}^{1}\right)^{2}+\left(\sigma_{22}^{1}\right)^{2}-\sigma_{11}^{1} \sigma_{22}^{1}+3\left(\sigma_{12}^{1}\right)^{2}$.

$\mathrm{K}$ полученным уравнениям необходимо добавить уравнения равновесия для приближений напряжений

$$
\sigma_{i j, j}^{m}=0, \quad m=1,2 .
$$

Если ввести функцию напряжений $F$ для приближений тензора напряжений с помощью формул

$$
\sigma_{i j, j}^{1}=\delta_{i j} \triangle F_{1}-F_{1, i j}, \quad \sigma_{i j, j}^{2}=\delta_{i j} \Delta F_{2}-F_{2, i j}
$$

$\left(\Delta F_{m}=F_{m, 11}+F_{m, 22}\right.$ - оператор Лапласа), то вместо системы четырёх уравнений (9)-(11) относительно напряжений $\sigma_{i j, j}^{m}$ получим два линейных уравнения относительно $F_{1}$ и $F_{2}$ :

$$
\begin{aligned}
& F_{1,1111}+F_{1,2222}+(3 n-1) F_{1,1122}=3 \sigma_{12}^{0} U, 12, \\
& F_{2,1111}+F_{2,2222}+(3 n-1) F_{2,1122}=\varphi\left(F_{1}, U\right) .
\end{aligned}
$$

Правая часть $\varphi\left(F_{1}, U\right)$ уравнения зависит от производных функций $F_{1}$ и $U$. В силу громоздкости здесь она не представлена.

Однородную функцию $U\left(x_{1}, x_{2}\right)$, с помощью которой даётся случайное поле возмущений реологических свойств материала, будем брать в виде [6]

$$
U\left(x_{1}, x_{2}\right)=A \cos \left(\omega_{1} x_{1}+\omega_{2} x_{2}+\varphi\right),
$$

где $A$ - центрированная случайная величина, $\varphi$ - случайная величина, имеющая равномерное распределение на интервале $(0 ; 2 \pi)$, причём $\left\langle A_{\varphi}\right\rangle=0, \omega_{1}$ и $\omega_{2}$ - положительные неслучайные параметры.

Для удобства выкладок целесообразно перейти к функции комплексной переменной

$$
\tilde{U}=\tilde{A} e^{i\left(\omega_{1} x_{1}+\omega_{2} x_{2}\right)},
$$

где $\tilde{A}=A e^{i \varphi}$. Функция $\tilde{U}$ введена так, что $\operatorname{Re} \tilde{U}=U$.

Будем рассматривать уравнения (13), (14), в которых действительные функции $U, F_{i}$ заменены комплексными $\tilde{U}, \tilde{F}_{i}$, причём $\operatorname{Re} \tilde{F}_{i}=F_{i}$.

Решение уравнения (13) имеет вид

$$
\tilde{F}_{1}=\tilde{A} f e^{i\left(\omega_{1} x_{1}+\omega_{2} x_{2}\right)}
$$

где $f$ - постоянная, найденная путём подстановки представлений $(16),(17)$ в уравнение (13):

$$
f=-\frac{3 \omega_{1} \omega_{2} \sigma_{12}^{0}}{\omega_{1}^{4}+\omega_{2}^{4}+(3 n-1) \omega_{1}^{2} \omega_{2}^{2}} .
$$


Выделяя действительную часть $\tilde{F}_{1}$, имеем

$$
F_{1}=\operatorname{Re} \tilde{F}^{1}=A f \cos \left(\omega_{1} x_{1}+\omega_{2} x_{2}+\varphi\right)
$$

или с учётом формулы $(15)-$

$$
F_{1}=f U\left(x_{1}, x_{2}\right)
$$

Используя соотношение (12), решение задачи в первом приближении в напряжениях можно записать в виде

$$
\begin{gathered}
\sigma_{11}^{(1)}=\alpha \sigma_{11}^{1}=-\alpha \omega_{2}^{2} f U=\frac{3 \alpha \omega_{1} \omega_{2}^{3} \sigma_{12}^{0} U}{\omega_{1}^{4}+\omega_{2}^{4}+(3 n-1) \omega_{1}^{2} \omega_{2}^{2}}, \\
\sigma_{22}^{(1)}=\alpha \sigma_{22}^{1}=-\alpha \omega_{1}^{2} f U=\frac{3 \alpha \omega_{1}^{3} \omega_{2} \sigma_{12}^{0} U}{\omega_{1}^{4}+\omega_{2}^{4}+(3 n-1) \omega_{1}^{2} \omega_{2}^{2}}, \\
\sigma_{12}^{(1)}=\sigma_{12}^{0}+\alpha \sigma_{12}^{1}=\sigma_{12}^{0}+\alpha \omega_{1} \omega_{2} f U=\sigma_{12}^{0}-\frac{3 \alpha \omega_{1}^{2} \omega_{2}^{2} \sigma_{12}^{0} U}{\omega_{1}^{4}+\omega_{2}^{4}+(3 n-1) \omega_{1}^{2} \omega_{2}^{2}} .
\end{gathered}
$$

Подставляя решение (17) в правую часть уравнения (14), можно получить

$$
\tilde{F}_{2,1111}+\tilde{F}_{2,2222}+(3 n-1) \tilde{F}_{2}, 1122=3 \tilde{A}^{2} b e^{2 i\left(\omega_{1} x_{1}+\omega_{2} x_{2}\right)},
$$

где $b=-2\left(36 k q f^{2} \omega_{1}^{3} \omega_{2}^{3}\left(\sigma_{12}^{0}\right)^{3}+4 k f^{2} \omega_{1} \omega_{2}\left(\omega_{1}^{2}+\omega_{2}^{2}\right)^{2} \sigma_{12}^{0}+6 k f^{2} \omega_{1} \omega_{2}\left(\omega_{1}^{4}+\omega_{2}^{4}\right) \sigma_{12}^{0}+\right.$ $\left.\left.+12 k f \omega_{1}^{2} \omega_{2}^{2}\right)^{2} \sigma_{12}^{0}+\left(\omega_{1}^{4}+\omega_{2}^{4}\right) f-\omega_{1} \omega_{2} \sigma_{12}^{0}\right)$.

Решение уравнения (19) можно искать в виде

$$
\tilde{F}_{2}=\tilde{A}^{2} g e^{2 i\left(\omega_{1} x_{1}+\omega_{2} x_{2}\right)},
$$

$g=$ const. После подстановки (20) в (19) для нахождения $g$ получается алгебраическое уравнение, из которого следует

$$
g=\frac{3 b}{16\left(\omega_{1}^{4}+\omega_{2}^{4}+(3 n-1) \omega_{1}^{2} \omega_{2}^{2}\right)} .
$$

Согласно (20) решение уравнения (19) определяется формулой

$$
\tilde{F}_{2}=\frac{3 \tilde{A}^{2} b e^{2 i\left(\omega_{1} x_{1}+\omega_{2} x_{2}\right)}}{16\left(\omega_{1}^{4}+\omega_{2}^{4}+(3 n-1) \omega_{1}^{2} \omega_{2}^{2}\right)} .
$$

Выделяя действительную часть $\tilde{F}_{2}$ и используя соотношения $(12)$, получим вторые приближения для компонент тензора напряжений:

$$
\begin{aligned}
\sigma_{11}^{2} & =-\frac{3 A^{2} b \omega_{2}^{2} \cos \left(2 \omega_{1} x_{1}+2 \omega_{2} x_{2}+\varphi\right)}{4\left(\omega_{1}^{4}+\omega_{2}^{4}+(3 n-1) \omega_{1}^{2} \omega_{2}^{2}\right)}, \\
\sigma_{22}^{2} & =-\frac{3 A^{2} b \omega_{1}^{2} \cos \left(2 \omega_{1} x_{1}+2 \omega_{2} x_{2}+\varphi\right)}{4\left(\omega_{1}^{4}+\omega_{2}^{4}+(3 n-1) \omega_{1}^{2} \omega_{2}^{2}\right)}, \\
\sigma_{12}^{2} & =\frac{3 \tilde{A}^{2} b \omega_{1} \omega_{2} \cos \left(2 \omega_{1} x_{1}+2 \omega_{2} x_{2}+\varphi\right)}{4\left(\omega_{1}^{4}+\omega_{2}^{4}+(3 n-1) \omega_{1}^{2} \omega_{2}^{2}\right)} .
\end{aligned}
$$


Решение задачи (1)-(3) во втором приближении согласно (5), (18), (21) определяется формулами

$$
\begin{gathered}
\sigma_{11}^{(2)}=\frac{3 \alpha \omega_{1} \omega_{2}^{3} \sigma_{12}^{0} U}{\omega_{1}^{4}+\omega_{2}^{4}+(3 n-1) \omega_{1}^{2} \omega_{2}^{2}}-\frac{3 A^{2} b \omega_{2}^{2} \cos \left(2 \omega_{1} x_{1}+2 \omega_{2} x_{2}+\varphi\right)}{4\left(\omega_{1}^{4}+\omega_{2}^{4}+(3 n-1) \omega_{1}^{2} \omega_{2}^{2}\right)}, \\
\sigma_{22}^{(2)}=\frac{3 \alpha \omega_{1}^{3} \omega_{2} \sigma_{12}^{0} U}{\omega_{1}^{4}+\omega_{2}^{4}+(3 n-1) \omega_{1}^{2} \omega_{2}^{2}}-\frac{3 A^{2} b \omega_{1}^{2} \cos \left(2 \omega_{1} x_{1}+2 \omega_{2} x_{2}+\varphi\right)}{4\left(\omega_{1}^{4}+\omega_{2}^{4}+(3 n-1) \omega_{1}^{2} \omega_{2}^{2}\right)}, \\
\sigma_{12}^{(2)}=\sigma_{12}^{0}-\frac{3 \alpha \omega_{1}^{2} \omega_{2}^{2} \sigma_{12}^{0} U}{\omega_{1}^{4}+\omega_{2}^{4}+(3 n-1) \omega_{1}^{2} \omega_{2}^{2}}+\frac{3 A^{2} b \omega_{1} \omega_{2} \cos \left(2 \omega_{1} x_{1}+2 \omega_{2} x_{2}+\varphi\right)}{4\left(\omega_{1}^{4}+\omega_{2}^{4}+(3 n-1) \omega_{1}^{2} \omega_{2}^{2}\right)} .
\end{gathered}
$$

Найдём основные статистические характеристики случайного поля напряжений: математическое ожидание и дисперсии компонент тензора напряжений. В силу того, что по условию задачи $\langle U\rangle=0$, имеем $\left\langle\sigma_{i j}^{(1)}\right\rangle=\left\langle\sigma_{i j}^{0}\right\rangle$.

Покажем, что $\left\langle\sigma_{i j}^{2}\right\rangle=0$. Для этого согласно $(21)$ достаточно установить, что $\left\langle A^{2} \cos \left(2 \omega_{1} x_{1}+2 \omega_{2} x_{2}+\varphi\right)\right\rangle=0$. В силу независимости случайных величин $A$ и $\varphi$ имеем $\left\langle\cos \left(2 \omega_{1} x_{1}+2 \omega_{2} x_{2}\right)+\varphi\right\rangle=\cos \left(2 \omega_{1} x_{1}+2 \omega_{2} x_{2}\right)\langle\cos \varphi\rangle+\sin \left(2 \omega_{1} x_{1}+\right.$ $\left.+2 \omega_{2} x_{2}\right)\langle\sin \varphi\rangle=0$. Поскольку случайная величина $\varphi$ распределена равномерно на отрезке $[0 ; 2 \pi]$, имеем $\langle\cos \varphi\rangle=0$ (см. [6]). Отсюда $\left\langle\sigma_{i j}^{2}\right\rangle=0$. Таким образом, математические ожидания в первом и втором приближениях равны $\sigma_{i j}^{0}$.

Дисперсии напряжений в первом приближении с учётом условия $\left\langle U^{2}\right\rangle=1$ определяются формулами

$$
\begin{aligned}
& D_{11}^{(1)}=\left\langle\left(\sigma_{11}^{1}\right)^{2}\right\rangle=\frac{9 \alpha^{2} \omega_{1}^{2} \omega_{2}^{6}\left(\sigma_{12}^{0}\right)^{2}}{\left(\omega_{1}^{4}+\omega_{2}^{4}+(3 n-1) \omega_{1}^{2} \omega_{2}^{2}\right)^{2}}, \\
& D_{22}^{(1)}=\left\langle\left(\sigma_{22}^{1}\right)^{2}\right\rangle=\frac{9 \alpha^{2} \omega_{1}^{6} \omega_{2}^{2}\left(\sigma_{12}^{0}\right)^{2}}{\left(\omega_{1}^{4}+\omega_{2}^{4}+(3 n-1) \omega_{1}^{2} \omega_{2}^{2}\right)^{2}}, \\
& D_{12}^{(1)}=\left\langle\left(\sigma_{12}^{1}\right)^{2}\right\rangle=\frac{9 \alpha^{2} \omega_{1}^{4} \omega_{2}^{4}\left(\sigma_{12}^{0}\right)^{2}}{\left(\omega_{1}^{4}+\omega_{2}^{4}+(3 n-1) \omega_{1}^{2} \omega_{2}^{2}\right)^{2}} .
\end{aligned}
$$

Дисперсии для второго приближения можно записать в виде

$$
D_{i j}^{(2)}=\alpha^{2} D\left[\sigma_{i j}^{1}\right]+\alpha^{4} D\left[\sigma_{i j}^{2}\right]+2 \alpha^{3}\left\langle\sigma_{i j}^{1} \sigma_{i j}^{2}\right\rangle .
$$

В дальнейшем предполагаем, что случайная величина $A$ распределена по нормальному закону. Для нормально распределённой случайной величины все центральные моменты нечётных порядков равны нулю, а момент четвёртого порядка $\left\langle A^{4}\right\rangle=3\left\langle A^{2}\right\rangle=6$ [7]. Момент $\left\langle\sigma_{i j}^{1} \sigma_{i j}^{2}\right\rangle$, входящий в (23), равен нулю, так как он пропорционален моменту третьего порядка $\left\langle A^{3}\right\rangle$. Дисперсия $D\left[\sigma_{i j}^{2}\right]$, как показано в работе [8], пропорциональна величине $0,5\left\langle A^{4}\right\rangle$.

Таким образом, дисперсии напряжений во втором приближении согласно (22), (23) определяются формулами

$$
D_{11}^{(2)}=\frac{9 \alpha^{2} \omega_{1}^{2} \omega_{2}^{6}\left(\sigma_{12}^{0}\right)^{2}}{\left(\omega_{1}^{4}+\omega_{2}^{4}+(3 n-1) \omega_{1}^{2} \omega_{2}^{2}\right)^{2}}+\frac{27 \alpha^{4} b^{2} \omega_{2}^{4}\left(\sigma_{12}^{0}\right)^{2}}{16\left(\omega_{1}^{4}+\omega_{2}^{4}+(3 n-1) \omega_{1}^{2} \omega_{2}^{2}\right)^{2}},
$$




$$
\begin{aligned}
& D_{22}^{(2)}=\frac{9 \alpha^{2} \omega_{1}^{6} \omega_{2}^{2}\left(\sigma_{12}^{0}\right)^{2}}{\left(\omega_{1}^{4}+\omega_{2}^{4}+(3 n-1) \omega_{1}^{2} \omega_{2}^{2}\right)^{2}}+\frac{27 \alpha^{4} b^{2} \omega_{1}^{4}\left(\sigma_{12}^{0}\right)^{2}}{16\left(\omega_{1}^{4}+\omega_{2}^{4}+(3 n-1) \omega_{1}^{2} \omega_{2}^{2}\right)^{2}}, \\
& D_{12}^{(2)}=\frac{9 \alpha^{2} \omega_{1}^{4} \omega_{2}^{4}\left(\sigma_{12}^{0}\right)^{2}}{\left(\omega_{1}^{4}+\omega_{2}^{4}+(3 n-1) \omega_{1}^{2} \omega_{2}^{2}\right)^{2}}+\frac{27 \alpha^{4} b^{2} \omega_{1}^{2} \omega_{2}^{2}\left(\sigma_{12}^{0}\right)^{2}}{16\left(\omega_{1}^{4}+\omega_{2}^{4}+(3 n-1) \omega_{1}^{2} \omega_{2}^{2}\right)^{2}} .
\end{aligned}
$$

Анализ случайного поля напряжений был проведён при условии, что частоты флуктуаций микронеоднородностей $\omega_{1}$ и $\omega_{2}$ относительно осей $x_{1}$ и $x_{2}$ равны между собой $\left(\omega_{1}=\omega_{2}=\omega\right)$. При этом условии случайное поле $U$, заданное разложением (15), можно считать близким к изотропному [1]. Дисперсии напряжений и в первом, и во втором приближениях будут равны между собой $\left(D_{11}=D_{22}=D_{12}=D\right)$ и не будут зависеть от $\omega$. Тогда формулы для вычисления коэффициента вариации будут иметь вид

$$
d_{1}=\frac{\sqrt{D^{(1)}}}{\sigma^{0}}=\frac{3 \alpha}{3 n+1}, \quad d_{2}=\frac{\sqrt{D^{(2)}}}{\sigma^{0}}=\frac{\sqrt{3} \alpha}{4(3 n+1)} \sqrt{48+\alpha^{2} b^{2}},
$$

где $\sigma^{0}=\sigma_{12}^{0}$.

Значения коэффициента вариации $d_{1}$ и $d_{2}$ (в процентах) как функции переменных $\alpha$ и $n$ приведены в табл. 1 . В столбце $d_{1}$ представлены значения коэффициента вариации, вычисленные по первому приближению, в столбце $d_{2}$ - по второму приближению. Как видно из табл. 1 , для материалов с высоким показателем нелинейности $(n=9)$ коэффициент вариации $d_{2}$ изменяется в пределах от $1,08 \%(\alpha=0,1)$ до $5,81 \%(\alpha=0,5)$. В случае низких показателей нелинейности, когда возможна полная физическая линеаризация закона ползучести $(n=1)$, разброс напряжений около среднего значения значительно больше: здесь значения коэффициента вариации заключены в пределах от $7,54 \%(\alpha=0,1)$ до $42,64 \%(\alpha=0,5)$.

В табл. 2 приведена относительная погрешность вычисления среднеквадратичного отклонения второго приближения относительно первого:

$$
\delta=\frac{\sqrt{D^{(2)}}-\sqrt{D^{(1)}}}{\sqrt{D^{(2)}}} \cdot 100 \% .
$$

Из табл. 2 видно, что имеется довольно обширная область значений параметров $\alpha$ и $n$, для которой погрешность $\delta<5 \%$ и первое (линейное) приближение вполне приемлимо для решения практических задач. Второе приближение необходимо использовать для материалов с большой степенью неоднородности $(\alpha=0,4 \div 0,5)$, особенно при малых значениях $n$.

Таблица 1

Значения коэффициента вариации $d_{1}$ и $d_{2}$ (в процентах)

\begin{tabular}{c|c|c|c|c|c|c|c|c|c|c}
\hline \multirow{2}{*}{$n$} & \multicolumn{2}{|c|}{1} & \multicolumn{2}{|c|}{3} & \multicolumn{2}{|c|}{5} & \multicolumn{2}{|c|}{7} & \multicolumn{2}{|c}{9} \\
\cline { 2 - 10 } & $d_{1}$ & $d_{2}$ & $d_{1}$ & $d_{2}$ & $d_{1}$ & $d_{2}$ & $d_{1}$ & $d_{2}$ & $d_{1}$ & $d_{2}$ \\
\hline 0,1 & 7,50 & 7,54 & 3,00 & 3,01 & 1,88 & 1,88 & 1,36 & 1,37 & 1,07 & 1,08 \\
0,2 & 15,00 & 15,35 & 6,00 & 6,09 & 3,75 & 3,80 & 2,73 & 2,77 & 2,14 & 2,17 \\
0,3 & 22,50 & 23,66 & 9,00 & 9,29 & 5,63 & 5,80 & 4,09 & 4,22 & 3,21 & 3,32 \\
0,4 & 30,00 & 32,69 & 12,00 & 12,67 & 7,50 & 7,91 & 5,45 & 5,75 & 4,29 & 4,52 \\
0,5 & 37,50 & 42,64 & 15,00 & 16,29 & 9,38 & 10,17 & 6,82 & 7,40 & 5,36 & 5,81 \\
\hline
\end{tabular}


Таблица 2

Относительная погрешность вычисления среднеквадратичного отклонения (в процентах)

\begin{tabular}{c|c|c|c|c|c|c|c|c|c}
\hline$\alpha$ & 1 & 2 & 3 & 4 & 5 & 6 & 7 & 8 & 9 \\
\hline 0,05 & 0,146 & 0,097 & 0,090 & 0,088 & 0,088 & 0,088 & 0,088 & 0,089 & 0,089 \\
0,10 & 0,581 & 0,388 & 0,358 & 0,351 & 0,350 & 0,351 & 0,352 & 0,353 & 0,354 \\
0,15 & 1,293 & 0,867 & 0,801 & 0,785 & 0,783 & 0,784 & 0,786 & 0,789 & 0,792 \\
0,20 & 2,264 & 1,526 & 1,410 & 1,383 & 1,379 & 1,381 & 1,385 & 1,390 & 1,395 \\
0,25 & 3,472 & 2,355 & 2,178 & 2,137 & 2,130 & 2,133 & 2,139 & 2,147 & 2,154 \\
0,30 & 4,890 & 3,339 & 3,092 & 3,034 & 3,024 & 3,029 & 3,038 & 3,049 & 3,059 \\
0,35 & 6,487 & 4,465 & 4,140 & 4,064 & 4,051 & 4,057 & 4,069 & 4,083 & 4,096 \\
0,40 & 8,234 & 5,717 & 5,308 & 5,212 & 5,196 & 5,204 & 5,219 & 5,236 & 5,253 \\
0,45 & 10,100 & 7,078 & 6,581 & 6,465 & 6,445 & 6,455 & 6,473 & 6,494 & 6,514 \\
0,50 & 12,056 & 8,531 & 7,945 & 7,808 & 7,785 & 7,796 & 7,818 & 7,842 & 7,867 \\
\hline
\end{tabular}

Работа выполнена при поддержке РФФИ (проект № 10-01-00644-а).

\section{БИБЛИОГРАФИЧЕСКИЙ СПИСОК}

1. Ломакин B. А. Статистические задачи механики твёрдых деформируемых тел. М.: Наука, 1970. 137 с. [Lomakin V.A. Statistical problems of the mechanics of deformable solids. Moscow: Nauka, 1970. 137 pp.]

2. Ломакин В.А. Проблемы механики структурно-неоднородных тел// Изв. АН СССР. $M T T$, 1978. № 6. C. 45-52. [Lomakin V. A. Problems in mechanics structurally nonuniform bodies // Izv. Akad. Nauk SSSR, Ser. Mekh. Tverd. Tela, 1978. no. 6. Pp. 45-52].

3. Попов Н. Н., Яшин М. А. Исследование случайных полей напряжений при чистом сдвиге стохастически неоднородной полуплоскости в условиях ползучести // Вестн. Сам. гос. техн. ун-та. Сер. Физ.-мат. науки, 2010. №1(20). С. 104-110. [Popov N. N., Yashin $M$. A. The study of random fields of stress in pure shear stochastically inhomogeneous halfplane under creep // Vestn. Samar. Gos. Tekhn. Univ. Ser. Fiz.-Mat. nauki, 2010. no. 1(20). Pp. 104-110].

4. Радченко В. П., Попов Н.Н. Стохастические характеристики полей напряжений и деформаций при установившейся ползучести стохастически неоднородной плоскости // Изв. вузов. Машиностроение, 2006. № 2. С. 3-11. [Radchenko V. P., Popov N. N. Stochastic characteristics of stress and strain fields in steady-state creep of stochastically inhomogeneous plane // Izv. Vuzov. Mashinostroenie, 2006. no. 2. Pp. 3-11].

5. Попов Н. Н., Коваленко Л. В., Яшин М. А. Решение плоской нелинейной стохастической задачи ползучести методом спектральных представлений// Beстн. Сам. гос. техн. унma. Сер. Физ.-мат. науки, 2009. № 2(19). С. 99-106. [Popov N. N.,Kovalenko L. V., Yashin $M$. A. Solution of plane nonlinear stochastic problem with spectral representation method // Vestn. Samar. Gos. Tekhn. Univ. Ser. Fiz.-Mat. nauki, 2009. no. 2(19). Pp. 99-106].

6. Вентцель Е. С., Овчаров Л. А. Прикладные задачи теории вероятностей. М.: Радио и связь, 1983. 416 с. [Wentzel E. S., Ovcharov L. A. Applied Problems in Probability Theory. Moscow: Radio i Svyaz', 1983. 416 pp.]

7. Пугачев В. С. Теория вероятностей и математическая статистика. М.: Физматлит, 2002. 496 c. [Pugachev V.S. Theory of Probability and Mathematical Statistics. Moscow: Fizmatlit, 2002. 496 pp.]

8. Попов Н. Н., Чернова О.О. Решение нелинейной задачи ползучести для стохастически неоднородной плоскости на основе второго приближения метода малого параметра // Вестн. Сам. гос. техн. ун-та. Сер. Физ.-мат. науки, 2011. №4(25). С. 50-58. [Ророv N. N., Chernova O. O. Solution of nonlinear creep problem for stochastically inhomogeneous 
plane on the basis of the second approximation for small parameter method // Vestn. Samar. Gos. Tekhn. Univ. Ser. Fiz.-Mat. nauki, 2011. no.4(25). Pp. 50-58].

Поступила в редакцию 01/XI/2012;

в окончательном варианте - 19/XI/2012.

MSC: 35Q74; 74E35, 74K20

METHOD OF PURE SHEAR PROBLEM SOLVING FOR

STOCHASTICALLY INHOMOGENEOUS PLANE IN A STEADY-STATE CREEP

\section{N. N. Popov, O. O. Chernova}

Samara State Technical University,

244, Molodogvardeyskaya st., Samara, 443100, Russia.

E-mails: ponick25@gmail.com, chernova_olga@citydom.ru

The analytical method for nonlinear problem of steady-state creep solving for pure shear of stochastically inhomogeneous plane on the basis of the second approximation method of small parameter was developed. It is supposed that elastic deformations are insignificant and they can be neglected. Stochasticity was introduced into the determinative creep equation, which was taken in accordance with the nonlinear theory of viscous flow, through a homogeneous random function of coordinates. By using the decomposition technique of stress tensor components in a small parameter to the members of the second order of smallness, partial differential system of the first and the second approximation of stress was obtained. This system was solved by the introduction of the stress function. The mathematical expectation and variances of the random stress field were calculated. The analysis of the results in the first and second approximations was obtained.

Key words: pure shear, small parameter method, steady-state creep, second approximation, stochastic problem.

Original article submitted $01 / \mathrm{XI} / 2012$; revision submitted 19/XI/2012.

Nikolay N. Popov (Ph. D. (Phys. \& Math.)), Associate Professor, Dept. of Applied Mathematics \& Computer Science.

Olga O. Chernova, Junior Research Scientist, Dept. of Applied Mathematics \& Computer Science. 